\title{
Understanding safety behaviour: Role of safety climate, supervisory behaviour, and individual motivation
}

Safety climate can be seen as a bridge between organizational rules/regulations and individual safety behaviour. But it is also important to examine how and through which mechanisms safety behaviour is manifested in employees. For this purpose, the present research aims to examine the relation between safety climate and safety behaviour, whether this relationship is mediated by motivations of workers to comply with the rules, and whether their relationship changes depending on supervisory behaviour. We collected data from 388 blue collar workers in a major factory. Analysis results show that safety climate is significantly related to safety behaviour of employees, and this relationship is partially mediated by motivations of workers to comply with the safety rules. Moreover, we have also tested the moderating role of supervisors' behavioural integrity for safety and found that workers reflect their motivations into actual behaviours more when their supervisors have higher behavioural integrity for safety. Findings indicate that organizational safety climate is associated with safety behaviours of workers both directly and through its association with safety motivations of workers. Moreover, the extent to which supervisors do what they preach amplifies the association of safety climate with safety motivation. These results contribute to workplace safety research by underscoring the importance of supervisor behavioural integrity in explaining under what circumstances the influence of safety climate on safety motivation is facilitated.

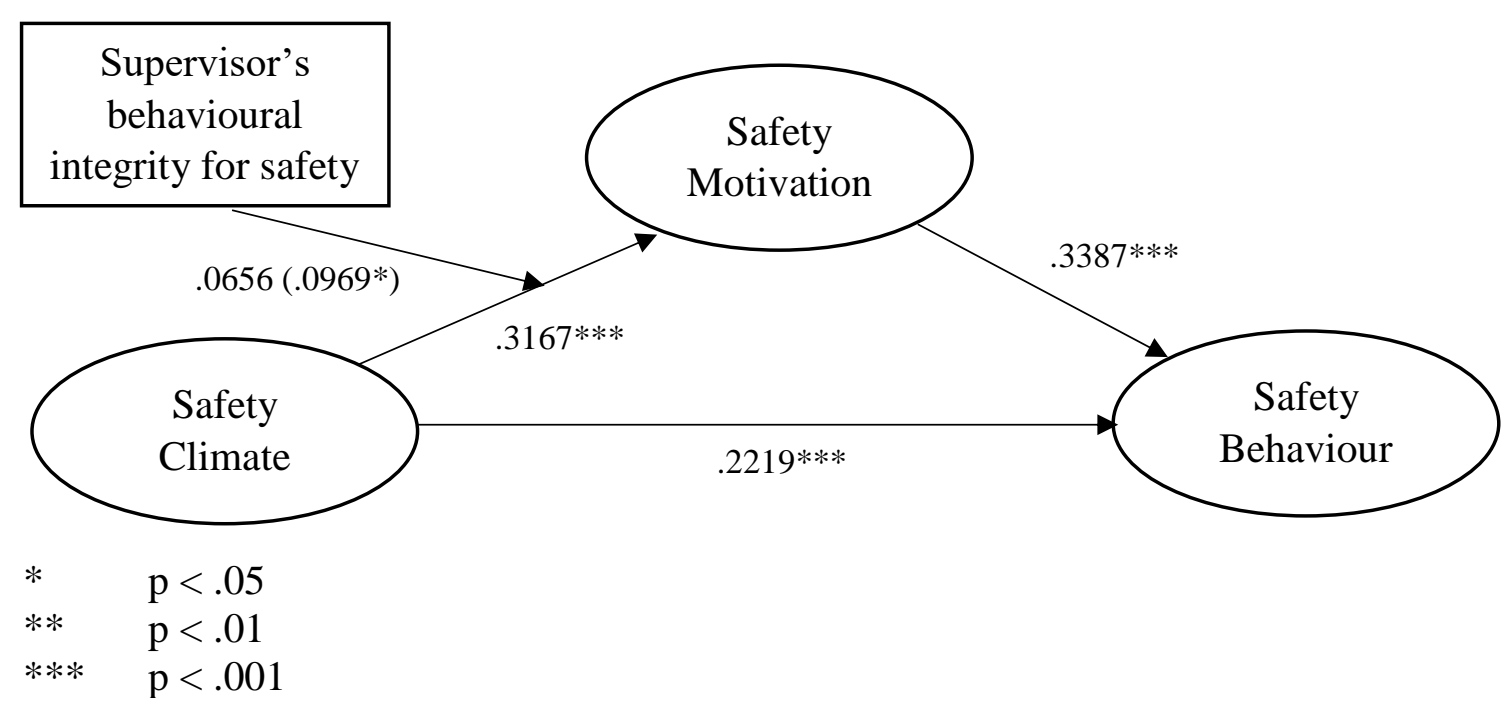


Run MATRIX procedure:

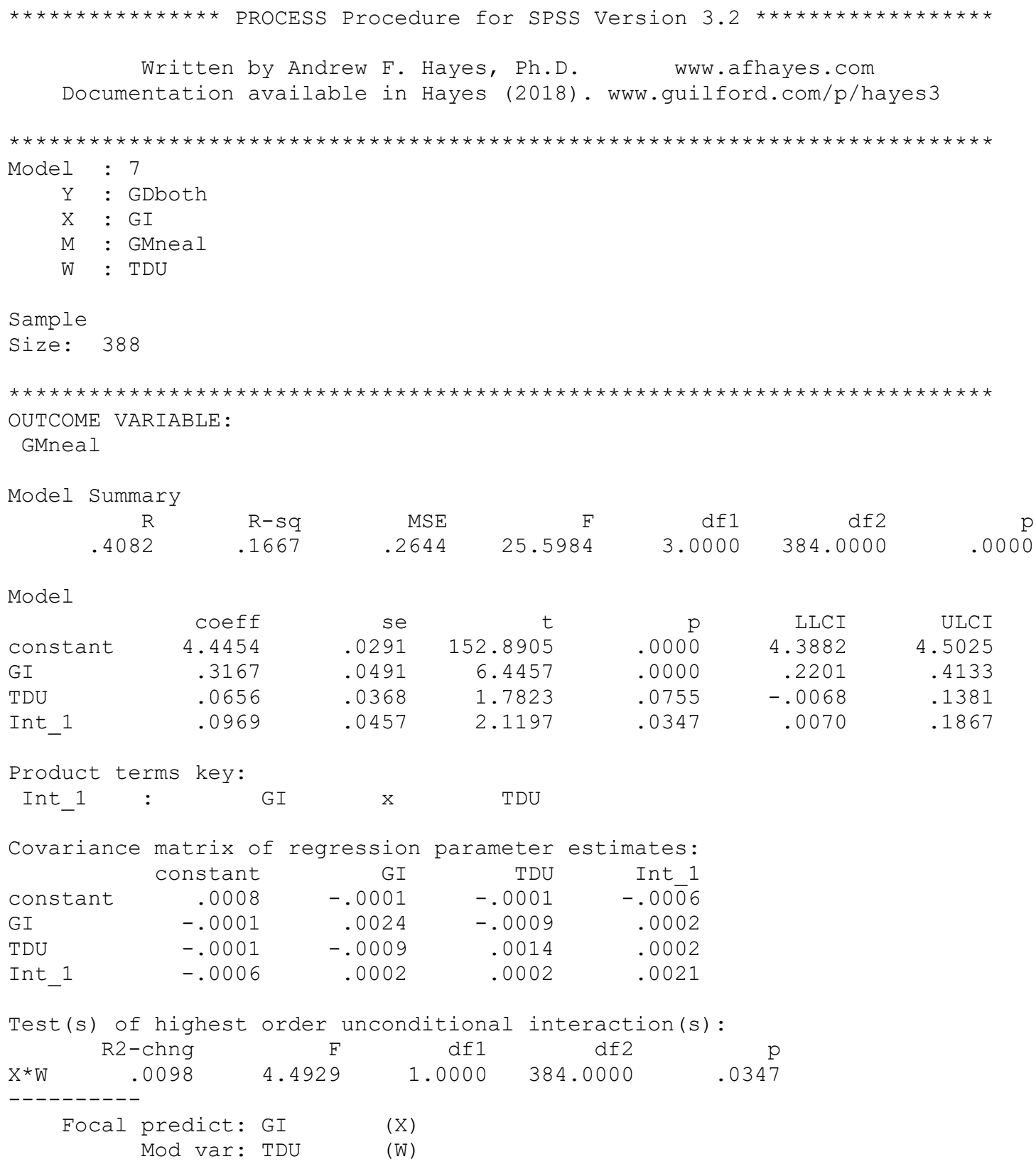

Model

$\begin{array}{lrrrrrr} & \text { coeff } & \text { se } & t & \text { P } & \text { LLCI } & \text { ULCI } \\ \text { Constant } & 4.4454 & .0291 & 152.8905 & .0000 & 4.3882 & 4.5025 \\ \text { GI } & .3167 & .0491 & 6.4457 & .0000 & .2201 & .4133 \\ \text { TDU } & .0656 & .0368 & 1.7823 & .0755 & -.0068 & .1381 \\ \text { Int_1 } & .0969 & .0457 & 2.1197 & .0347 & .0070 & .1867\end{array}$

Product terms key:

Int_1 : GI $\quad$ x $\quad$ TDU

Covariance matrix of regression parameter estimates:

$\begin{array}{lrrrr} & \text { constant } & \text { GI } & \text { TDU } & \text { Int_1 } \\ \text { constant } & .0008 & -.0001 & -.0001 & -.0006 \\ \text { GI } & -.0001 & .0024 & -.0009 & .0002 \\ \text { TDU } & -.0001 & -.0009 & .0014 & .0002 \\ \text { Int_1 } & -.0006 & .0002 & .0002 & .0021\end{array}$

Test(s) of highest order unconditional interaction(s):

$\begin{array}{rrrrrr} & R 2-\operatorname{chng} & \mathrm{F} & \mathrm{df1} & \mathrm{df2} & \mathrm{p} \\ \mathrm{X} * \mathrm{~W} & .0098 & 4.4929 & 1.0000 & 384.0000 & .0347\end{array}$

Focal predict: GI (X) Mod var: TDU (W)

Conditional effects of the focal predictor at values of the moderator(s):

\begin{tabular}{|c|c|c|c|c|c|c|}
\hline TDU & Effect & $\mathrm{se}$ & t & $\mathrm{p}$ & LLCI & ULCI \\
\hline-.7314 & .2459 & .0567 & 4.3348 & .0000 & .1344 & .357 \\
\hline .1019 & .3266 & .0498 & 6.5582 & .0000 & .2287 & \\
\hline .9352 & .4073 & .0681 & 5.9774 & .0000 & .2733 & .54 \\
\hline
\end{tabular}

Data for visualizing the conditional effect of the focal predictor:

Paste text below into a SPSS syntax window and execute to produce plot.

DATA LIST FREE/

GI TDU

BEGIN DATA.

$$
\begin{array}{rrr}
-.6210 & -.7314 & 4.2447 \\
.1077 & -.7314 & 4.4238 \\
.6077 & -.7314 & 4.5468 \\
-.6210 & .1019 & 4.2492 \\
.1077 & .1019 & 4.4872 \\
.6077 & .1019 & 4.6505
\end{array}
$$

GMneal 


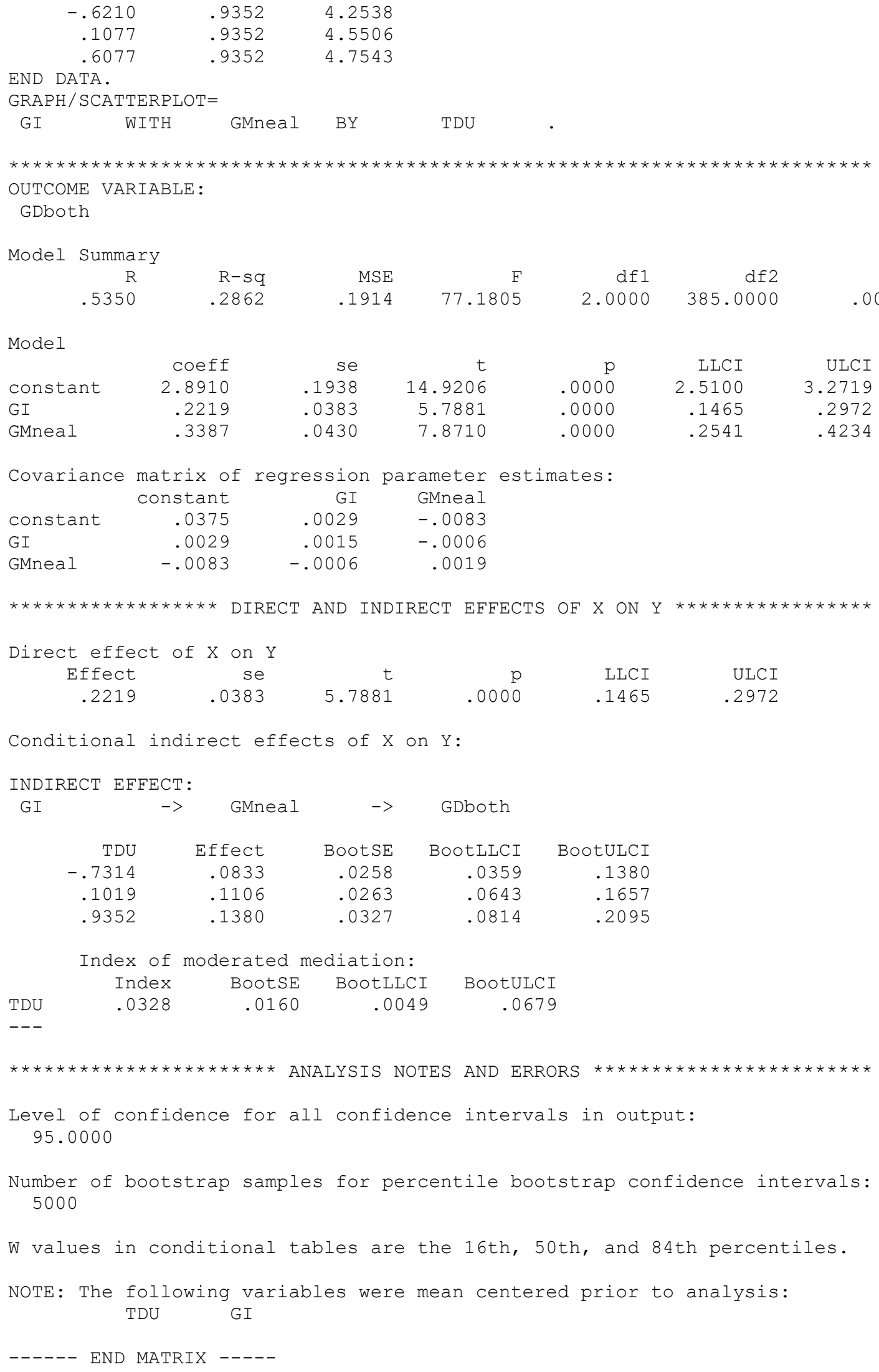

Model

$\begin{array}{lrrrrrr} & \text { coeff } & \text { se } & t & p & \text { LLCI } & \text { ULCI } \\ \text { Constant } & 2.8910 & .1938 & 14.9206 & .0000 & 2.5100 & 3.2719 \\ \text { GI } & .2219 & .0383 & 5.7881 & .0000 & .1465 & .2972 \\ \text { GMneal } & .3387 & .0430 & 7.8710 & .0000 & .2541 & .4234\end{array}$

Covariance matrix of regression parameter estimates:

$\begin{array}{lrrr} & \text { constant } & \text { GI } & \text { GMneal } \\ \text { Constant } & .0375 & .0029 & -.0083 \\ \text { GI } & .0029 & .0015 & -.0006 \\ \text { GMneal } & -.0083 & -.0006 & .0019\end{array}$

$\begin{array}{rrrrrr}\text { Effect } & \text { se } & t & p & \text { LLCI } & \text { ULCI } \\ .2219 & .0383 & 5.7881 & .0000 & .1465 & .2972\end{array}$

Conditional indirect effects of $\mathrm{X}$ on $\mathrm{Y}$ :

INDIRECT EFFECT:

$->$

GMneal $\quad->\quad$ GDboth

$\begin{array}{rrrrr}\text { TDU } & \text { Effect } & \text { BootSE } & \text { BootLLCI } & \text { BootULCI } \\ -.7314 & .0833 & .0258 & .0359 & .1380 \\ .1019 & .1106 & .0263 & .0643 & .1657 \\ .9352 & .1380 & .0327 & .0814 & .2095\end{array}$

Index of moderated mediation:

$\begin{array}{rrrrr} & \text { Index } & \text { BootSE } & \text { BootLLCI } & \text { BootULCI } \\ \text { TDU } & .0328 & .0160 & .0049 & .0679\end{array}$


Introduction

Organizational climate is reported to affect individual behaviour. Defined as the individual perceptions of organization's stand in safety related policies, procedures, and practices; safety climate is not an exception in influencing individual safety behaviour. It is well established that safety climate is associated with positive outcomes in safety related issues such as safety participation, safety compliance, and decreased overall accidents/injuries (Clarke, 2006). Note that despite being similar, safety culture and safety climate should be distinguished. Schein (1992) suggests that climate precedes culture or, in other words, climate manifests creation of a certain culture. We believe that using safety climate, rather than culture, is more suitable in organizational settings.

Obviously, being in a workplace where it is a social norm to prioritize safety issues improves compliance with the safety rules. But "how" this relationship is established or formed is the main focus of our study. There are studies suggesting that individuals' motivation to exert the behaviours plays a mediating role between safety climate and safety behaviour. Studies indicate that safety climate in organizations increases safety motivations of individuals which in turn increases safety behaviour (Neal, Griffin, \& Hart, 2000; Neal \& Griffin, 2006). We wanted to explore this relationship when supervisory approaches are also included in the equation. Specifically, we wanted to test if (1) the extent to which supervisors do what they preach moderates the relationship between safety climate and safety motivation, and if the mediation of motivation between climate and behaviour is true for a Turkish sample.

\section{Method}

Participants - We collected data from 376 blue collar workers working in a domestic appliances factory in Turkey, as part of a safety assessment project. The participants were from 18 different departments with different shifts such as exchange, goods receiving, montage, etc. The participants were predominantly male with $91 \%$ of the total sample. Average age for the participants was 32.5 years $(\mathrm{SD}=7.64)$, and average tenure for the participants was 6.95 years $(\mathrm{SD}=7.01)$.

Measures - Along with a short demographic form, participants were asked to fill short questionnaires about (1) organizational-level safety climate, (2) behavioural integrity for safety, (3) safety motivation, and (4) safety behaviour.

Data Analysis - We used Hayes Macro (Model 7) for moderated mediation in SPSS (25.0).

Procedure and Hypothesis - The organization contacted us in demand for a safety assessment project. We took ethical approval from Ege University and collected the data at the beginning of this assessment. Our primary hypothesis was that (1) safety motivation will mediate the relationship between safety climate and safety behaviour. Our secondary hypothesis was that (2) workers with supervisors high in behavioural integrity about safety will be more motivated when they perceive a better safety climate.

Results

Analysis results yielded significant relationship between both variables (see Fig. 1). As expected Moderated mediation analysis with the current variables explained $16.67 \%$ variance in safety motivation, and $28.62 \%$ variance in safety behaviour. Safety motivation partially mediated the relationship between safety climate and safety behaviour, as this relationship was significant even without the safety motivation. Moreover, supervisor's behavioural integrity moderated the relationship between safety climate and safety motivation, with higher integrity yielding stronger relationship between these variables. So, it can be suggested that our hypothesis were supported.

\begin{tabular}{|l|l|l|l|l|l|}
\hline Table 1. Standardized effects: Mediation model & \multicolumn{4}{l|}{} \\
\hline & Stand. Coef. & S.E. & $\mathrm{p}$ & LLCI 95 \% & ULCI 95\% \\
\hline SC --> SM & .3167 & .0491 & .0000 & .2201 & .4133 \\
\hline SBI --> SM & .0656 & .0368 & .0755 & -.0068 & .1381 \\
\hline
\end{tabular}




\begin{tabular}{|l|l|l|l|l|l|}
\hline SM --> SB & .2219 & .0383 & .0000 & .1465 & .2972 \\
\hline SC --> SB & .3387 & .0430 & .0000 & .2541 & .4234 \\
\hline & Indirect Effect & & & & \\
\hline SC --> SB & $? ? ?$ & $? ? ?$ & $? ? ?$ & $? ? ?$ & $? ? ?$ \\
\hline & \multicolumn{5}{|l|}{} \\
\hline $\begin{array}{l}\text { SC: Safety Climate, SM: Safety Motivation, SBI: Supervisor's Behavioural Integrity for Safety, SB: } \\
\text { Safety Behaviour }\end{array}$
\end{tabular}

Table 1. Standardized effects: Mediation model

\begin{tabular}{llllll}
\hline & Stand. Coef. & S.E. & p & LLCI 95 \% & ULCI 95 \% \\
\hline SC --> SM & .32 & .05 & $<.001$ & .22 & .41 \\
SBI --> SM & .07 & .04 & .08 & -.01 & .14 \\
SM --> SB & .22 & .04 & $<.001$ & .15 & .30 \\
SC --> SB & .34 & .04 & $<.001$ & .25 & .42 \\
\hline
\end{tabular}

SC: Safety Climate, SM: Safety Motivation, SBI: Supervisor's Behavioural Integrity for Safety, SB: Safety Behaviour

\begin{tabular}{|l|l|l|l|l|l|}
\hline Table 2. Standardized effects: Moderation model \\
\hline & Stand. Coef. & S.E. & p & LLCI 95 \% & ULCI 95 \% \\
\hline SBI -1 SD & .2459 & .0567 & .0000 & .1344 & .3574 \\
\hline SBI &. .3266 & .0498 & .0000 & .2287 & .4245 \\
\hline SBI +1 SD & .4073 & .0681 & .0000 & .2733 & .5413 \\
\hline SBI: Supervisor's Behavioural Integrity for Safety \\
\hline
\end{tabular}

\section{Discussion}

In the current study, we replicated previous findings (Neal \& Griffin, 2000) suggesting the mediating role of safety motivation between one's perceptions about organizations cumulative opinions and one's own behaviour. We believe that replicating this finding with a Turkish sample was required. We have also contributed to explanation of this relationship with

\section{Introduction}

Organizational climate is reported to affect individual behaviour. Defined as the individual perceptions of organization's stand in safety related policies, procedures, and practices; safety climate is not an exception in influencing individual safety behaviour. It is well established that safety climate is associated with positive outcomes in safety related issues such as safety participation, safety compliance, and decreased overall accidents/injuries (Clarke, 2006).

Obviously, being in a workplace where it is a social norm to prioritize safety issues improves compliance with the safety rules. But "how" this relationship is established or formed is the main focus of our study. There are studies suggesting that individuals' motivation to exert the behaviours plays a mediating role between safety climate and safety behaviour. Studies indicate that safety climate in organizations increases safety motivations of individuals which in turn increases safety behaviour (Neal, Griffin, \& Hart, 2000; 
Neal \& Griffin, 2006). We wanted to explore this relationship when supervisory approaches are also included in the equation.

Method

Participants

376 blue collar workers

Predominantly male sample (91\%)

Mean age 32.5 years $(\mathrm{SD}=7.64)$

Mean tenure 6.95 years $(\mathrm{SD}=7.01)$

Measures

Organizational-level safety climate

Supervisory behavioural integrity for safety

Safety motivation

Safety behaviour

Procedure

The organization contacted us in demand for a safety assessment project. We took ethical approval from Ege University and collected the data at the beginning of this assessment.

Results

Analysis results yielded significant relationship between variables (see Fig. 1). As expected moderated mediation analysis with the current variables explained $16.67 \%$ variance in safety motivation, and $28.62 \%$ variance in safety behaviour. Safety motivation partially mediated the relationship between safety climate and safety behaviour. Moreover, supervisor's behavioural integrity moderated the relationship between safety climate and safety motivation.

Discussion

In the current study, we replicated previous findings (Neal \& Griffin, 2000) suggesting the mediating role of safety motivation between one's perceptions about organizations stand and one's behaviour in a Turkish sample. Our unique contribution to safety research is adding the moderation of supervisory integrity about safety related issues.

Table 1. Standardized effects: Mediation model

\begin{tabular}{llllll}
\hline & Stand. Coef. & S.E. & p & LLCI 95 \% & ULCI 95 \% \\
\hline SC $\rightarrow$ SM & .3167 & .0491 & .0000 & .2201 & .4133 \\
SBI $\rightarrow$ SM & .0656 & .0368 & .0755 & -.0068 & .1381 \\
SM $\rightarrow$ SB & .2219 & .0383 & .0000 & .1465 & .2972 \\
SC $\rightarrow$ SB & .3387 & .0430 & .0000 & .2541 & .4234 \\
& Indirect & & & & \\
& Effect & & & & \\
\hline SC $\rightarrow$ SB & $? ? ?$ & $? ? ?$ & $? ? ?$ & $? ? ?$ & $? ? ?$ \\
\hline
\end{tabular}


SC: Safety Climate, SM: Safety Motivation, SBI: Supervisor's Behavioural Integrity for Safety, SB: Safety Behaviour 